\title{
Curcumin Increase the Expression of Neural Stem/Progeni- tor Cells and Improves Functional Recovery after Spinal Cord Injury
}

\author{
Woo-Seok Bang, M.D., ${ }^{1}$ Kyoung-Tae Kim, M.D., Ye Jin Seo, M.S., ${ }^{1}$ Dae-Chul Cho, M.D., Joo-Kyung Sung, M.D., ${ }^{1}$ Chi Heon Kim, M.D. ${ }^{2}$ \\ Department of Neurosurgery, Kyungpook National University Hospital, Daegu, Korea \\ Department of Neurosurgery, ${ }^{2}$ Seoul National University Hospital, Seoul, Korea
}

Objective : To investigates the effect of curcumin on proliferation of spinal cord neural stem/progenitor cells (SC-NSPCs) and functional outcome in a rat spinal cord injury (SCl) model.

Methods : Sixty adult male Sprague-Dawley rats were randomly and blindly allocated into three groups (sham control group; curcumin treated group after $\mathrm{SCl}$; vehicle treated group after $\mathrm{SCl}$ ). Functional recovery was evaluated by the Basso, Beattie, and Bresnahan (BBB) scale during 6 weeks after SCl. The expression of SC-NSPC proliferation and astrogliosis were analyzed by nestin/Bromodeoxyuridine (BrdU) and Glial fibrillary acidic protein (GFAP) staining. The injured spinal cord was then examined histologically, including quantification of cavitation.

Results : The BBB score of the SCl-curcumin group was better than that of $\mathrm{SCl}$-vehicle group up to 14 days $(p<0.05)$. The coimmunoreactivity of nestin/BrdU in the SCl-curcumin group was much higher than that of the SCl-vehicle group 1 week after surgery $(p<0.05)$. The GFAP immunoreactivity of the SCl-curcumin group was remarkably lower than that of the SCl-vehicle group 4 weeks after surgery $(p<0.05)$. The lesion cavity was significantly reduced in the curcumin group as compared to the control group $(p<0.05)$.

Conclusion : These results indicate that curcumin could increase the expression of SC-NSPCs, and reduce the activity of reactive astrogliosis and lesion cavity. Consequently curcumin could improve the functional recovery after SCI via SC-NSPC properties.

Key Words : Curcumin · Spinal cord injuries · Neural stem progenitor cells · Lesion cavity · Astrogliosis · Functional recovery.

\section{INTRODUCTION}

Traumatic spinal cord injury (SCI) is a clinically irreversible condition and results in catastrophic disability. Acute SCI has two physiological cascading mechanisms ${ }^{2,3)}$. The pathophysiology of acute SCI is complex and occurs via two mechanisms, involving a primary and secondary injury. The primary injury is the initial structural damage caused by a mechanical trauma. The second injury is a subsequent step after the primary injury and can cause a progressive apoptosis caused by local inflammation, production of free radicals, and hyperoxida$\operatorname{tion}^{5,12,37)}$. Among these mechanisms contributing to secondary injury, inflammatory reactions bring out the reactive astrogliosis, and this reaction produces the glial scar which can

- Received : March 8, 2017 • Revised : May 12, 2017 •Accepted : May 31, 2017

-Address for reprints : Kyoung-Tae Kim, M.D.

Department of Neurosurgery, Kyungpook National University Hospital, 130 Dongdeok-ro, Jung-gu, Daegu 41944, Korea Tel : +82-53-420-5657, Fax : +82-53-423-0504, E-mail : nskimkt7@gmail.com

This is an Open Access article distributed under the terms of the Creative Commons Attribution Non-Commercial License (http://creativecommons.org/licenses/by-nc/4.0) which permits unrestricted non-commercial use, distribution, and reproduction in any medium, provided the original work is properly cited. 
be a physical barrier for neuronal regeneration after $\mathrm{SCI}^{300}$. These cascading mechanisms make recovery from SCI hard or impossible. Many methods have been attempted to manage SCI so far. However, there are no fully effective methods. Thus, now in medical science, researching the most effective cure methods for SCI is a crucial task. Stem cell therapy is one of the currently-studied research programs.

According to previous studies, neural stem/progenitor cells (NSPCs) exist in the human adult stage as well as fetal stage ${ }^{11,15)}$. Moreover, recent studies reported that SCI could induce expression of NSPCs, and resulting NSPCs were related to functional and histologic recoveries after $\mathrm{SCI}^{8,14,25,29)}$. Stem cell therapy for SCI is focused on clinical usage of NSPCs' biological activity.

Curcumin (diferuloylmethane) (1,7-bis(4-hydroxy-3-methoxyphenyl)-1,6-heptadiene-3,5-dione) is a natural ingredient, which has been used as an Indian spice. So far, much research revealed that curcumin has numerous biologic effects, including anti-inflammatory, antioxidant effects ${ }^{10,12,24,27,31,33,34,39)}$. Recent study using a fetal rat brain revealed that curcumin promoted neurogenesis ${ }^{17)}$. However, there have been no in vivo studies to date to reveal whether curcumin affects differentiation and proliferation of spinal cord NSPCs (SC-NSPCs) in the SCI condition.

To date, the NPSC expression, astrogliosis, lesion cavity, and functional recovery effects of curcumin have not been fully evaluated. Therefore, the aim of the present study is to evaluate the effect of curcumin on the expression of SCNSPCs, reactive astrogliosis, lesion cavity, and functional recovery after SCI.

\section{MATERIALS AND METHODS}

\section{Animal population and experimental design}

All animal experiments were performed in accordance with the National Institute of Health guidelines on animal care, and were approved by the Institutional Animal Care Committee of our institute. All efforts were made to minimize the number of animals used and animal suffering. Animals were housed in a temperature-controlled room on a 12-hour light/ dark circadian cycle.

\section{Culture of SC-NSPC}

To establish cultures of SC-NSPCs, Sprague-Dawley rats weighting $250 \mathrm{~g}$ to $350 \mathrm{~g}$ were sacrificed and their spinal cords were extracted. The spinal cords were chopped using microscissors and incubated in a cocktail containing papain $(0.1 \%$; Worthington Biochemical Corp., Lakewood Township, NJ, USA), dispase (0.1\%), DNase (0.01\%), and $\mathrm{MgSO}_{4}(12.4 \%)$ in a Hanks balanced salt solution with glucose $(0.45 \%)$ for $30 \mathrm{~min}$ utes at $37^{\circ} \mathrm{C}$. The dissociated cells were cultured to form neurospheres in a neurobasal medium containing B-27, glutamin $(2 \mathrm{mmol} / \mathrm{L})$, penicillin/streptomycin $(0.1 \mathrm{~g} / \mathrm{mL})$, fibroblast growth factor-2 $(20 \mathrm{ng} / \mathrm{mL})$, and heparin $(2 \mu \mathrm{g} / \mathrm{mL})$. After 7 days, the neurospheres were dissociated with Accutase (Innovative Cell Technologies Inc., San Diego, CA, USA), and $5 \times 10^{5}$ NSPCs were plated in plates or dishes containing $5 \mathrm{~mL}$ of the culture medium. The NSPCs between 5 and 25 passages were used for the experiments.

\section{SC-NSPC proliferation assay}

The NSPCs $\left(2 \times 10^{4}\right)$ were suspended in $100 \mathrm{~mL}$ of a neurobasal medium containing various concentrations of curcumin $(0.1-30 \mu \mathrm{mol} / \mathrm{L})$ and cultured in a $96-w e l l$ plate for 3 days. A $20-\mathrm{mL}$ cell proliferation assay solution containing the tetrazolium compound 3-(4,5-dimethylthiazol-2-yl)-5-(3carboxymethoxyphenyl)-2-(4-sulfophenyl)-2H-tetrazolium (MTS, CellTiter 96 Aqueous One Solution; Promega, Madison, WI, USA) was added to the cultured cells and incubated for 2.5 hours at $37^{\circ} \mathrm{C}$. The optical density was measured at $490 \mathrm{~nm}$ with a microplate spectrophotometer.

\section{$\mathrm{SCl}$ models}

Total sixty adult male Sprague-Dawley rats weighing 290$310 \mathrm{~g}$ (Samtako Bio, Osan, Korea) were randomly and blindly allocated into three groups ( $\mathrm{n}=20$ per group). In sham group, only laminectomy was performed without SCI. SCI-curcumin group animals were given a SCI and received $1 \mu \mathrm{mol} / \mathrm{L}$ of curcumin (Sigma-Aldrich, St. Louis, MO, USA) once a day by intrathecal injection. SCI-vehicle group animals were treated with the vehicle only by intrathecal injection following SCI.

The surgical technique used for SCI in rats has been previously described by the authors ${ }^{16,38)}$. Rats were anesthetized via intraperitoneal injection of a mixture of xylazine $(10 \mathrm{mg} / \mathrm{kg})$ and ketamine $(60 \mathrm{mg} / \mathrm{kg})$. After laminectomy at T9, the extra- 
dural plane between the dura and the adjacent vertebrae was carefully dissected. A modified aneurysm clip with a closing force of $30 \mathrm{~g}$ (Aesculap, Tuttlingen, Germany) was held in an applicator in the open position. The clip was rapidly released from the applicator and applied vertically onto the exposed spinal cord for a 2-minute compression. For the sham controls, the same surgical procedure was followed, but clip compression was not applied. After surgery, the muscle, fascia, and skin were sutured using a $4-0$ silk suture. The rectal temperature was maintained at $37.0 \pm 0.5^{\circ} \mathrm{C}$ by a thermostatically-regulated heating pad during surgery, and during recovery, animals were placed overnight in a temperature- and humidity-controlled chamber. To reduce post-surgery isolation-induced stress, rats were housed in pairs at an ambient temperature of $22-25^{\circ} \mathrm{C}$ in an alternating 12 -hour light/dark cycle. Bladders were manually emptied twice daily until spontaneous voiding occurred (usually within 7-10 days).

\section{Administration of curcumin}

The method of constructing and implanting indwelling intrathecal catheters was described previously ${ }^{16,23}$. Briefly, immediately after SCI or the sham operation, intrathecal catheters were implanted into rats under anesthesia by threading a sterile polyethylene-10 catheter guided by a 20 -gauge needle between the L5-6 vertebrae. The catheter was inserted rostrally into the subdural space of the spinal cord. The curcumin were reconstituted in artificial cerebrospinal fluid (ACSF) (119 mmol/L NaCl, $3.1 \mathrm{mmol} / \mathrm{L} \mathrm{KCl}, 1.2 \mathrm{mmol} / \mathrm{L} \mathrm{CaCl}_{2}, 1$ $\mathrm{mmol} / \mathrm{L} \mathrm{MgSO}_{4}, 0.50 \mathrm{mmol} / \mathrm{L} \mathrm{KH}_{2} \mathrm{PO}_{4}, 25 \mathrm{mmol} / \mathrm{L} \mathrm{NaHCO}_{3}$, $5 \mathrm{mmol} / \mathrm{L} \mathrm{D}$-glucose, and $2.2 \mathrm{mmol} / \mathrm{L}$ urea, $\mathrm{pH} 7.4$ ) at a concentration of $1 \mu \mathrm{mol} / \mathrm{L}$ for curcumin. The control was filled with ACSF alone. Both the compounds were infused for 7 days at a rate of $10 \mu \mathrm{L}$ per day. To label the proliferating NSPCs, rats were intraperitoneally injected with bromodeoxyuridine (BrdU) $(50 \mathrm{mg} / \mathrm{kg}$ in saline; Sigma-Aldrich) twice a day for 7 days starting 1 day after the intrathecal injection.

\section{Analysis of newly proliferated SC-NSPC}

At 1 week after SCI, six rats from each of the three groups were deeply anesthetized by an intraperitoneal injection of ketamine and were intracardially perfused with $4 \%$ paraformaldehyde in $0.1 \mathrm{M}$ sodium phosphate buffer ( $\mathrm{PB} ; \mathrm{pH}=7.4$ ). The thoracic spinal cord was excised, postfixed for 24 hours, and maintained overnight in 30\% sucrose in $0.1 \mathrm{M}$ phosphate buffer solution. Spinal cord tissues were sectioned at a thickness of $30 \mu \mathrm{m}$ on a cryostat, and sections were floated on the surface of $0.1 \mathrm{M}$ phosphate buffer solution. A 5 and $6 \mathrm{~mm}$ rostral to the center of injury was performed. To detect nestin (a marker for NSPCs), spinal cord sections were blocked with $4 \%$ normal serum in $0.5 \%$ Triton X-100 for 1 hour at room temperature, incubated overnight at $4{ }^{\circ} \mathrm{C}$ with a $1: 200$ dilution of mouse monoclonal anti-nestin (R\&D Systems, Inc., Minneapolis, MN, USA) and rabbit polyclonal anti-BrdU (Abcam, Cambridge, MA, USA), and then rinsed for three times for 10 minutes in a $0.1 \mathrm{M} \mathrm{PB}$. Sections were incubated for 2 hours with Alexa Fluor 594 goat anti-mouse IgG and Alexa Fluor 488 goat anti-rabbit IgG (both $1: 300$; invitrogen). The images were viewed on a computer monitor using a Zeiss Plan-Apochromat 40X objective (Carl Zeiss Meditec Inc., Jena, Germany). Enumeration of immune-positive cells used a Labworks, version 4.5, computer-assisted image analyzer (UVP, Upland, CA, USA).

\section{Analysis of peri-lesional astrogliosis}

At 4 weeks after SCI, six rats from each of the three groups were sacrificed. Thoracic cord was excised and treated in the same method for analysis of newly proliferated SC-NSPC. A 5 and $6 \mathrm{~mm}$ rostral to the center of injury was performed. To detect anti-Glial fibrillary acidic protein (GFAP) (marker for astrocyte), spinal cord sections were blocked. A $1: 200$ dilution of mouse monoclonal anti-GFAP (Abcam) was used. Method of immune-positive cells enumeration is same as assay for newly proliferated SC-NSPC.

\section{Measurement of lesion cavity volume}

Six weeks after SCI, eight rats from each of the two groups (SCI-vehicle and SCI-curcumin) were sacrificed. A $1.5 \mathrm{~cm}$ segment of the spinal cord centered at the injury site was immediately harvested from the vertebral canal and postfixed in $10 \%$ formalin overnight. The portion of the spinal cord was divided into seven segments ( $4 \mathrm{~mm}$ and $2 \mathrm{~mm}$ rostral to the lesion; lesion epicenter; $2 \mathrm{~mm}$ and $4 \mathrm{~mm}$ caudal to the lesion) at $2 \mathrm{~mm}$ intervals from the lesion epicenter. Representative sections were sliced into $5 \mu \mathrm{m}$-thick sections on the transverse plane and stained with hematoxylin-eosin. To quantitative evaluate of lesion cavity areas, 20 sequential slides of the serial 
sections were obtained from representative segments. The tissues were examined and photographed using a Zeiss Axioplan microscope (Carl Zeiss Meditec Inc.). The areas of lesion cavity were traced and measured using Axio-Vision 4 software (Carl Zeiss Meditec Inc.).

\section{Locomotor and behavior analyses}

The rats were tested for functional deficits for 6 weeks after the surgery using the open field locomotor rating scale developed by the Basso, Beattie, and Bresnahan (BBB) score ${ }^{4)}$. Two evaluators who were unaware of the group allocations and previous functional scores observed each animal for 1 minute. Functional scores for each hind limb were recorded and averaged.

\section{Statistical analysis}

All statistical comparisons were computed using SPSS version 17.0 (SPSS Inc., Chicago, IL, USA). Data are expressed as mean \pm standard deviation of the mean. Repeated measure analysis of variance was used to compare groups. Significance was accepted for $p$ values $<0.05$.

\section{RESULTS}

\section{Decision of curcumin concentration to increases the proliferation of SC-NSPCs}

To determine the proper concentration of curcumin, SCNSPCs growing in 96-well plates were maintained in medium lacking or containing various concentrations of curcumin, and the cell proliferation rate was quantified at different time points. After 72 hours of curcumin treatment, curcumin has biphasic effects on SC-NSPC proliferation. Curcumin of 1 $\mu \mathrm{mol} / \mathrm{L}$ has most effective in proliferation of NSPC, whereas high dosage ( $\geq 5 \mu \mathrm{mol} / \mathrm{L})$ caused a decrease in NSPC proliferation (Fig. 1).

\section{Newly expressed NSPC at 1 week after SCI}

To understand whether curcumin can promote NSPC expression in spinal cord, we infused $1 \mu \mathrm{mol} / \mathrm{L}$ curcumin into the subarachnoid space of adult rats for 7 days after SCI and treated them intraperitoneally with BrdU. The area of the Nestin+/BrdU+ cells in the SCI-curcumin group (183.7 \pm 11.5$)$ was significantly increased compared to that in the SCI-vehi- cle group (97.3 \pm 5.4$) 1$ week after surgery (Fig. 2). This result indicates that curcumin could stimulate the expression of SCNSPCs after SCI.

\section{Peri-lesional astrogliosis at 4 weeks after SCI}

To evaluate whether curcumin can influence the astrogliosis, we checked the GFAP (astrocyte marker). The area of GFAP was remarkably higher in the SCI-vehicle group (69730 \pm 1403$)$ compare with the SCI-curcumin group $(8034 \pm 155) 4$ weeks after surgery (Fig 3). This result suggests that curcumin would influence the astrogliosis after SCI.

\section{Lesion cavities}

Six weeks following SCI, histological examination revealed a central cavity with peri-lesional regeneration. The lesion cavity extended to over $2 \mathrm{~mm}$ rostrally and $2 \mathrm{~mm}$ caudally, tapering gradually to cavities affecting the central and dorsal areas of the spinal cord gray and white matter (Fig. 4). In SCIcurcumin group, the area of the lesion cavity was significantly decrease compared to that of the rats that received vehicle solution only $(p<0.05)$. Also, we could find neurogenesis in perilesional area.

\section{Locomotor and behavior analysis}

The injured rats were assessed for 6 weeks after surgical-

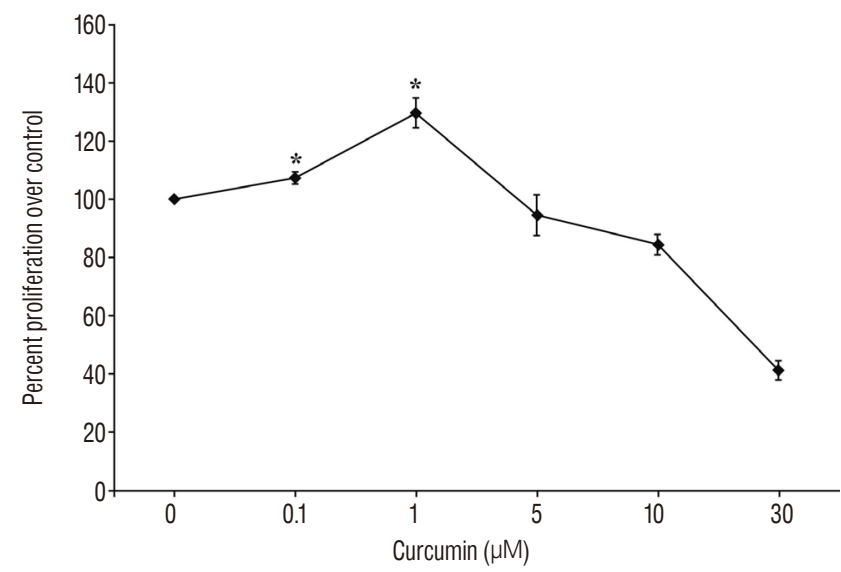

Fig. 1. Curcumin has biphasic effects on SC-NSPC proliferation. The SCNSPC proliferation rate was quantified at different time points. After 72 hours of curcumin treatment, lower curcumin dosage $(0.1$ and $1 \mu \mathrm{mol} / \mathrm{L})$ showed significant increase of NSPC proliferation. But higher dosage $(5$, 10 , and $30 \mu \mathrm{mol} / \mathrm{L}$ ) of curcumin decreased the NSPC proliferation rate. *Significantly increased compared with corresponding value for control group $(p<0.05)$. NSPC : neural stem/progenitor cell, SC-NSPC : spinal cord NSPC. 

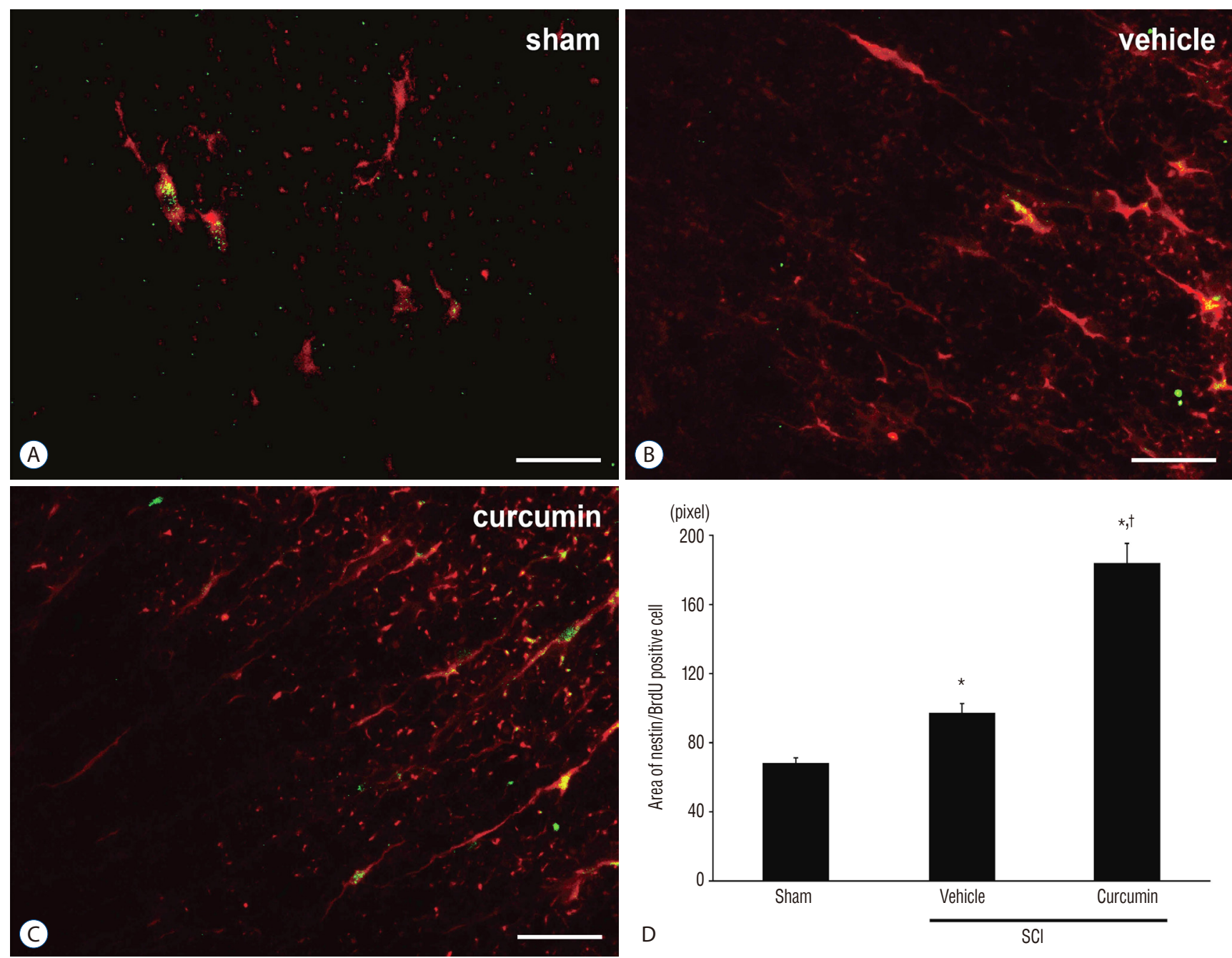

Fig. 2. Fluoroscopic images demonstrating newly expressed neural stem/progenitor cells (co-expression of BrdU and Nestin). A : Sham group (Immunofluorescence, $\times 20$ ). B : SCl-vehicle group (Immunofluorescence, $\times 20$ ). C : SCl-curcumin group (Immunofluorescence, $\times 20$ ). D : The bar graph reveals the quantification of cells positive for both $\mathrm{BrdU}$ and nestin in the spinal cord. Values in the histogram represent the means \pm standard deviation. ${ }^{*} p<0.05$, compared with the respective sham control. ${ }^{\dagger} p<0.05$, compared with the respective vehicle control. Bars $=50 \mu \mathrm{m}$. BrdU : Bromodeoxyuridine, $\mathrm{SCl}$ : spinal cord injury.

ly-induced SCI according to open filed motor testing using the BBB score. While all rats exhibited severe functional impairment following SCI, the motor function of the curcumin-injected rats was markedly better than the vehicle-injected rats 14 days after SCI, which was statistically significant $(p<0.05)$ (Fig. 5). This significance was maintained during 6 weeks and functional improvement was not observed after 3 weeks.

\section{DISCUSSION}

Traumatic SCI typically leads to permanent neurological deficits in motor and sensory systems. However, all the general methods, including high-dose steroid therapy, early decompressive surgery, and long-standing rehabilitation, have not been a sufficient solution for recovery from SCI. Inoue reported that axonal regeneration occurred beyond the transection site in young rats and resulted in good functional improvement ${ }^{13)}$. Recently, the proliferation and neuronal differentiation of NSPC after SCI played an important role in functional recovery after $\mathrm{SCI}^{14,19,25)}$.

Curcumin's potentiality to increase the proliferation of NSPCs was already reported in previous studies ${ }^{1,1722,40)}$. We already reported that curcumin stimulated proliferation of SC- 

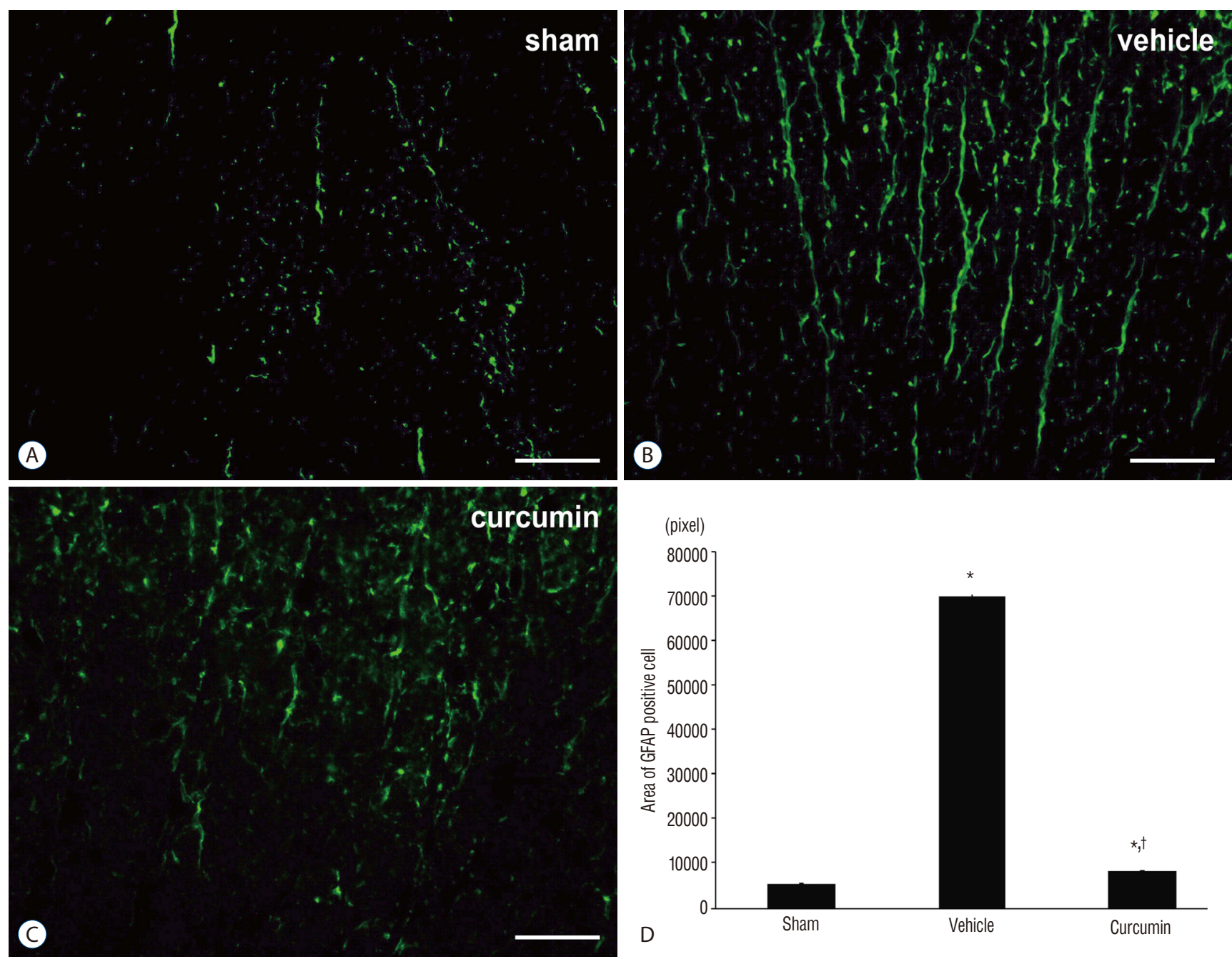

Fig. 3. Fluoroscopic images demonstrating the area of astrogliosis at 2 weeks after SCl. A : Sham group (Immunofluorescence, $\times 20$ ). B : SCl-vehicle group (Immunofluorescence, $\times 20$ ). C : SCl-curcumin group (Immunofluorescence, $\times 20$ ). D : The bar graph reveals the quantification of Glial fibrillary acidic protein positive in the spinal cord. Values in the histogram represent the means \pm standard deviation. ${ }^{*} p<0.05$, compared with the respective sham control. ${ }^{\dagger} p<0.05$, compared with the respective vehicle control. Bars $=50 \mu \mathrm{m} . \mathrm{SCl}$ : spinal cord injury.

NSPCs through a MAP kinase signaling pathway ${ }^{35)}$. In an extension of our study, we sought to investigate the effect of curcumin on expression of SC-NSPCs and activity of astrogliosis, and functional recovery after SCI in this study. The finding that the proliferation degree of NSPCs depends on dosage of curcumin was verified by recent studies ${ }^{7,28,36)}$. In other words, the proper dosage of curcumin for proliferation of NSPCs has a threshold value. However, the subject of the above studies was not SC-NSPCs. To decide the proper dosage, we used an SC-NSPC proliferation assay, and active SCNSPC proliferation in the curcumin group compared to the control group was observed in concentrations below $1 \mu \mathrm{mol} / \mathrm{L}$ of curcumin (Fig. 1). In concentrations over $5 \mu \mathrm{mol} / \mathrm{L}$ of cur- cumin, SC-NSPCs proliferation in the curcumin group sharply decreased with higher concentrations. After work, we administrated $1 \mu \mathrm{mol} / \mathrm{L}$ of curcumin to mice by intrathecal injection every day for 1 week, and after we used immunohistochemistry of nestin \& BrdU to evaluate the proliferation of SC-NSPCs by curcumin. According to the study of Shibuya et al. $^{29)}$, we set an evaluation timing of immunofluorescence analysis as 1 week after surgery, because nestin expression peaked at 1 week after surgery in their study. The result of this study showed an increased area of nestin $+/ \mathrm{Brdu}+$ cells in the SCI-curcumin group.

In the study of Kim et al., ${ }^{17)}$ hippocampal neurogenesis was observed in curcumin-administrated adult mice. More re- 

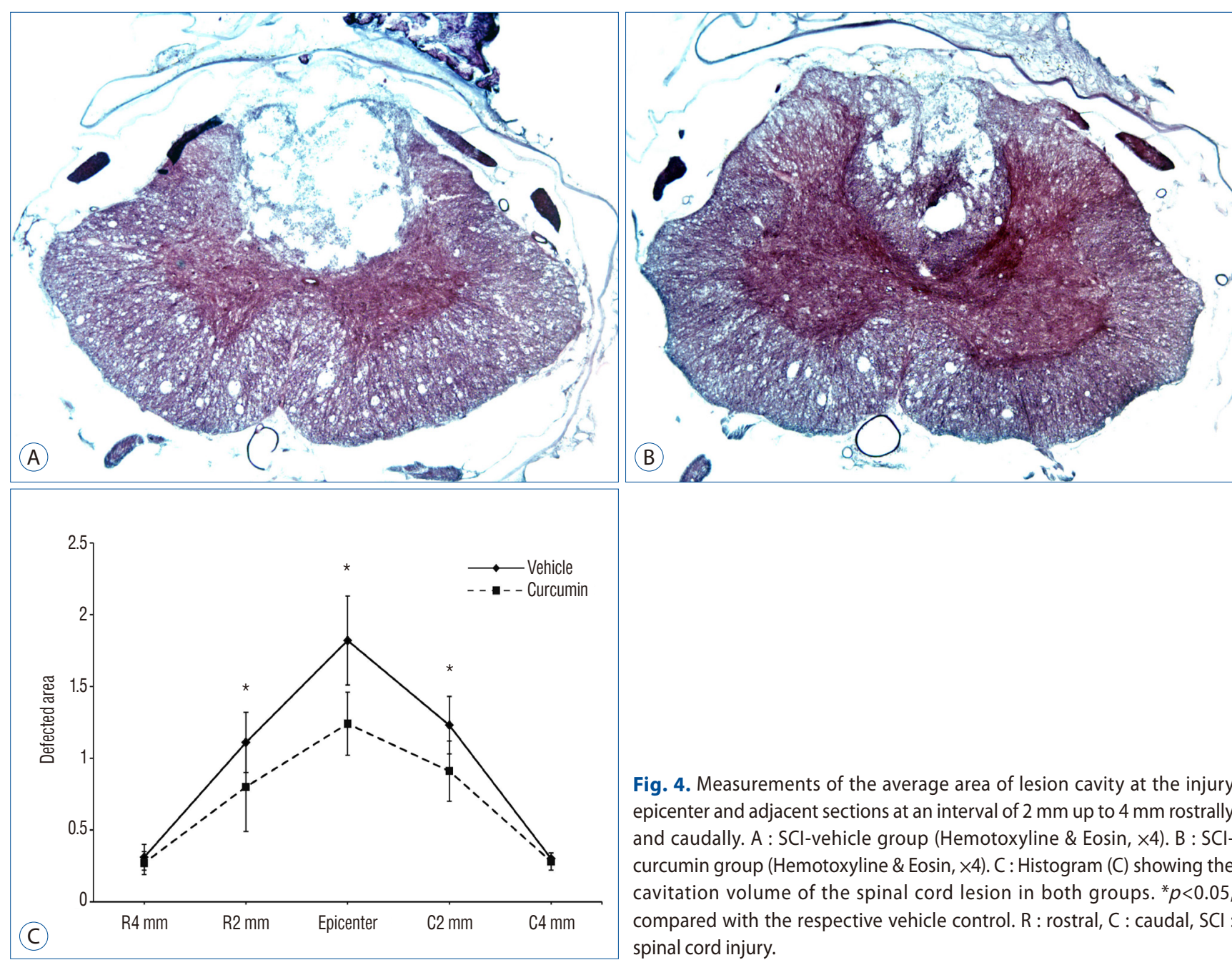

Fig. 4. Measurements of the average area of lesion cavity at the injury epicenter and adjacent sections at an interval of $2 \mathrm{~mm}$ up to $4 \mathrm{~mm}$ rostrally and caudally. A : SCl-vehicle group (Hemotoxyline \& Eosin, $\times 4$ ). B : SCIcurcumin group (Hemotoxyline \& Eosin, $\times 4$ ). C : Histogram (C) showing the cavitation volume of the spinal cord lesion in both groups. ${ }^{*} p<0.05$, compared with the respective vehicle control. $\mathrm{R}$ : rostral, $\mathrm{C}$ : caudal, $\mathrm{SCl}$ : spinal cord injury.

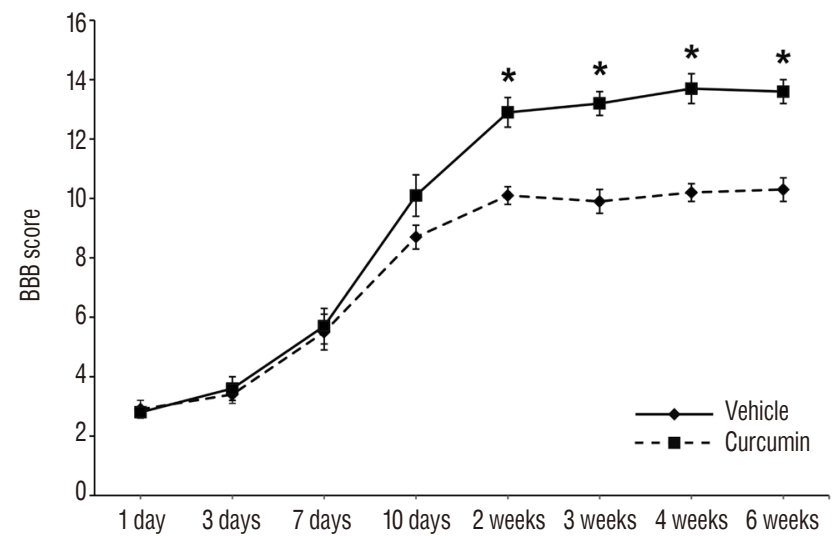

Fig. 5. Graph showing locomotor rating scale by BBB scores. BBB score of the $\mathrm{SCl}$-curcumin group was significantly better than the vehicleinjected rats after 14 days $\mathrm{SCl}$. *Indicates $p<0.05$, compared with the respective vehicle control. $\mathrm{BBB}$ : Basso, Beattie, and Bresnahan, $\mathrm{SCl}$ : spinal cord injury. cently, in the study of Chen et al. ${ }^{7)}$ using two-thirds of the fetal rat forebrain, differentiation of neural stem cells was checked in the curcumin group. They explained this result in virtue of curcumin's property to activate the Wnt signaling pathway. In the present study, we used a rat SCI model and observed the decrease of lesion cavity volume (Fig. 4). Also, for the result of BBB score of the entire period, the SCI-curcumin group showed a statistically better motor function than the SCI-vehicle group. Based on these results, we can guess that facilitated proliferation of SC-NSPCs and peri-lesional regeneration induced by curcumin contribute to functional recovery from SCI.

To discuss regeneration in the damaged spinal cord, we cannot help but mention reactive astrogliosis. Astrocytes play a big role as a primary regulator of homeostasis and supporting cells in the normal central nervous system $(\mathrm{CNS})^{9,26)}$. 
Curcumin Promotes Functional Recovery | Bang WS, et al.

However, in the damaged spinal cord, astrocytes show somewhat different activity in comparison with the normal CNS. After SCI, reactive astrogliosis, which was triggered by proinflammatory cytokines such as TNF- $\alpha$, IL- $1 \beta$, and IL-6, arises and this phenomenon changes the damaged spinal cord into a more difficult environment for neurogenesis ${ }^{18,20)}$. Glial scars created by reactive astrogliosis make axonal regeneration difficult as a physical barrier ${ }^{30)}$. Besides, reactive astrocytes secrete the extracellular inhibitory substances, including chondroitin sulfate proteoglycan (CSPG), and axonal regeneration can't proceed in CSPG-rich regions ${ }^{6,32)}$. In Lin's study, the reduction of GFAP expression in the SCI-curcumin group was demonstrated $^{21)}$. Their study means that curcumin can inhibit the reactive astrogliosis after SCI. Furthermore, Chen et al. ${ }^{6)}$ reported various roles for curcumin in neuroprotection after SCI. They proved not only decreased expression of GFAP, but decreased expression of proinflammatory cytokines and decreased deposition of CSPG by curcumin. Our study also showed strongly decreased GFAP stain in the SCI-curcumin group compared to the SCI-vehicle group at 4 weeks after surgery (Fig. 3). Considering the lesion cavity and regeneration result of the SCI-curcumin group at 6 weeks after surgery, we can build a reasonable hypothesis that attenuated reactive astrogliosis provides a good circumstance for regeneration after SCI.

\section{CONCLUSION}

This study demonstrated that curcumin could promote the expression of SC-NSPCs, and reduce the activity of reactive astrogliosis and lesion cavity. Considering these results, we can suggest that curcumin could improve the functional recovery after SCI via SC-NSPC properties.

\section{PATIENT CONSENT}

The patient provided written informed consent for the publication and the use of their images.

\section{- Acknowledgements}

This research was supported by Basic Science Research Program through the National Research Foundation of Korea (NRF) funded by the Ministry of Science, ICT \& Future Plan- ning (NRF-2015R1C1A1A01056299).

\section{References}

1. Alvarez-Buylla A, García-Verdugo JM, Tramontin AD : A unified hypothesis on the lineage of neural stem cells. Nat Rev Neurosci 2 : 287293, 2001

2. Balentine JD : Pathology of experimental spinal cord trauma. I. The necrotic lesion as a function of vascular injury. Lab Invest 39 : 236-253, 1978

3. Balentine JD : Pathology of experimental spinal cord trauma. II. Ultrastructure of axons and myelin. Lab Invest 39 : 254-266, 1978

4. Basso DM, Beattie MS, Bresnahan JC : Graded histological and locomotor outcomes after spinal cord contusion using the NYU weight-drop device versus transection. Exp Neurol 139 : 244-256, 1996

5. Bethea JR, Dietrich WD : Targeting the host inflammatory response in traumatic spinal cord injury. Curr Opin Neurol 15 : 355-360, 2002

6. Chen DY, Lu XH, Chen Y, Yan WJ, Yang HS, Wang XW, et al. : Anterior decompression for the treatment of cervical spondylotic myelopathy associated with ossification of posterior longitudinal ligament. Zhonghua Wai Ke Za Zhi 47 : 610-612, 2009

7. Chen F, Wang H, Xiang X, Yuan J, Chu W, Xue X, et al. : Curcumin increased the differentiation rate of neurons in neural stem cells via wnt signaling in vitro study. J Surg Res 192 : 298-304, 2014

8. Chen Z, Liu B, Dong J, Feng F, Chen R, Xie P, et al. : Comparison of anterior corpectomy and fusion versus laminoplasty for the treatment of cervical ossification of posterior longitudinal ligament: a meta-analysis. Neurosurg Focus 40 : E8, 2016

9. Dong Y, Benveniste EN : Immune function of astrocytes. Glia 36 : 180190, 2001

10. Folwarczna J, Zych M, Trzeciak HI : Effects of curcumin on the skeletal system in rats. Pharmacol Rep 62 : 900-909, 2010

11. Gage FH, Coates PW, Palmer TD, Kuhn HG, Fisher LJ, Suhonen JO, et al. : Survival and differentiation of adult neuronal progenitor cells transplanted to the adult brain. Proc Natl Acad Sci U S A 92 : 1187911883, 1995

12. Houle JD, Tessler A : Repair of chronic spinal cord injury. Exp Neurol $182: 247-260,2003$

13. Inoue $T$, Kawaguchi $S$, Kurisu $K$ : Spontaneous regeneration of the pyramidal tract after transection in young rats. Neurosci Lett $247: 151$ 154, 1998

14. Iwai H, Nori S, Nishimura S, Yasuda A, Takano M, Tsuji O, et al. : Transplantation of neural stem/progenitor cells at different locations in mice with spinal cord injury. Cell Transplant 23 : 1451-1464, 2014

15. Johansson CB, Momma S, Clarke DL, Risling $M$, Lendahl U, Frisén J : Identification of a neural stem cell in the adult mammalian central nervous system. Cell 96 : 25-34, 1999

16. Kim KT, Kim HJ, Cho DC, Bae JS, Park SW : Substance P stimulates proliferation of spinal neural stem cells in spinal cord injury via the mitogen- 
activated protein kinase signaling pathway. Spine J 15 : 2055-2065, 2015

17. Kim SJ, Son TG, Park HR, Park M, Kim MS, Kim HS, et al. : Curcumin stimulates proliferation of embryonic neural progenitor cells and neurogenesis in the adult hippocampus. J Biol Chem 283 : 14497-14505, 2008

18. Kordek R, Nerurkar VR, Liberski PP, Isaacson S, Yanagihara R, Gajdusek $D C$ : Heightened expression of tumor necrosis factor alpha, interleukin 1 alpha, and glial fibrillary acidic protein in experimental creutzfeldt-jakob disease in mice. Proc Natl Acad Sci U S A 93 : 9754-9758, 1996

19. Li J, Lepski $G$ : Cell transplantation for spinal cord injury: a systematic review. Biomed Res Int 2013 : 786475, 2013

20. Lin HW, Basu A, Druckman C, Cicchese M, Krady JK, Levison SW : Astrogliosis is delayed in type 1 interleukin-1 receptor-null mice following a penetrating brain injury. J Neuroinflammation 3 : 15, 2006

21. Lin MS, Lee YH, Chiu WT, Hung KS : Curcumin provides neuroprotection after spinal cord injury. J Surg Res 166 : 280-289, 2011

22. McNally SJ, Harrison EM, Ross JA, Garden OJ, Wigmore SJ : Curcumin induces heme oxygenase 1 through generation of reactive oxygen species, p38 activation and phosphatase inhibition. Int J Mol Med 19 : 165-172, 2007

23. Milligan ED, Hinde JL, Mehmert KK, Maier SF, Watkins LR : A method for increasing the viability of the external portion of lumbar catheters placed in the spinal subarachnoid space of rats. J Neurosci Methods $90: 81-86,1999$

24. Moon Y, Glasgow WC, Eling TE : Curcumin suppresses interleukin 1betamediated microsomal prostaglandin E synthase 1 by altering early growth response gene 1 and other signaling pathways. J Pharmacol Exp Ther 315 : 788-795, 2005

25. Nakamura $\mathrm{M}, \mathrm{Okano} \mathrm{H}$ : Cell transplantation therapies for spinal cord injury focusing on induced pluripotent stem cells. Cell Res $23: 70-80$, 2013

26. Nicoll JA, Weller RO : A new role for astrocytes: beta-amyloid homeostasis and degradation. Trends Mol Med 9 : 281-282, 2003

27. Sahin Kavaklı H, Koca C, Alıcı 0 : Antioxidant effects of curcumin in spinal cord injury in rats. Ulus Travma Acil Cerrahi Derg 17 : 14-18, 2011

28. Shehzad A, Lee YS : Molecular mechanisms of curcumin action: signal transd uction. Biofactors 39 : 27-36, 2013

29. Shibuya S, Miyamoto O, Auer RN, Itano T, Mori S, Norimatsu H : Embryonic intermediate filament, nestin, expression following traumatic spinal cord injury in adult rats. Neuroscience 114 : 905-916, 2002

30. Shih $\mathrm{CH}$, Lacagnina $\mathrm{M}$, Leuer-Bisciotti $\mathrm{K}$, Pröschel $\mathrm{C}$ : Astroglial-derived periostin promotes axonal regeneration after spinal cord injury. J Neurosci 34 : 2438-2443, 2014

31. Shishodia S, Sethi $G$, Aggarwal BB : Curcumin: getting back to the roots. Ann N Y Acad Sci 1056 : 206-217, 2005

32. Silver J, Miller JH : Regeneration beyond the glial scar. Nat Rev Neurosci 5 : 146-156, 2004

33. Soetikno V, Sari FR, Veeraveedu PT, Thandavarayan RA, Harima M, Sukumaran $V$, et al. : Curcumin ameliorates macrophage infiltration by inhibiting NF-kB activation and proinflammatory cytokines in streptozotocin induced-diabetic nephropathy. Nutr Metab (Lond) 8 : 35, 2011

34. Somasundaram S, Edmund NA, Moore DT, Small GW, Shi YY, Orlowski RZ : Dietary curcumin inhibits chemotherapy-induced apoptosis in models of human breast cancer. Cancer Res 62 : 3868-3875, 2002

35. Son S, Kim KT, Cho DC, Kim HJ, Sung JK, Bae JS : Curcumin stimulates proliferation of spinal cord neural progenitor cells via a mitogen-activated protein kinase signaling pathway. J Korean Neurosurg Soc 56 : 1-4, 2014

36. Suh HW, Kang S, Kwon KS: Curcumin attenuates glutamate-induced HT22 cell death by suppressing MAP kinase signaling. Mol Cell Biochem 298 : 187-194, 2007

37. Tator $\mathrm{CH}$, Fehlings MG : Review of the secondary injury theory of acute spinal cord trauma with emphasis on vascular mechanisms. J Neurosurg $75: 15-26,1991$

38. Wells JE, Hurlbert RJ, Fehlings MG, Yong VW : Neuroprotection by minocycline facilitates significant recovery from spinal cord injury in mice. Brain 126(Pt 7) : 1628-1637, 2003

39. Wu A, Ying Z, Gomez-Pinilla F : Dietary curcumin counteracts the outcome of traumatic brain injury on oxidative stress, synaptic plasticity, and cognition. Exp Neurol 197 : 309-317, 2006

40. Zhang $X$, Yin WK, Shi XD, Li Y : Curcumin activates Wnt/ $\beta$-catenin signaling pathway through inhibiting the activity of GSK-3 $\beta$ in APPswe transfected SY5Y cells. Eur J Pharm Sci 42 : 540-546, 2011 